\title{
Binocular coordination of saccades: development, aging and cerebral substrate
}

\author{
Zoï Kapoula \\ Iris Laboratory, FRE 3154, CNRS \\ Qing Yang \\ Iris Laboratory, FRE 3154, CNRS
}

\author{
Marine Vernet \\ Iris Laboratory, FRE 3154, CNRS \\ Maria Pia Bucci \\ Iris Laboratory, FRE 3154, CNRS
}

\begin{abstract}
The origin of binocular coordination of saccades (central, peripheral) and the role of learning remain controversial (Hering vs Helmholtz). We will present evidence for learning: in young children (5 years) horizontal saccades are poorly yoked, coordination improves slowly with age particularly at near viewing distances. In dyslexic teenagers coordination of horizontal saccades is poor relative to non-dyslexics, suggesting slower learning. On the other hand, in healthy elderly participants ( 73 years) coordination of vertical saccades is intact, an example of a non ageing sub-system. To assess further central mechanisms, we applied TMS over the posterior parietal cortex of healthy adults, $100 \mathrm{~ms}$ after target onset. TMS impaired coordination particularly for rightward and downward saccades. Thus binocular coordination of saccades relies partially on cerebral function.
\end{abstract}

Keywords: binocular coordination, saccades, children, dyslexic, elderly, TMS

\section{Hering-Helmholtz controversy: a reconciling hypothesis}

Fine binocular coordination of saccades is essential for clear binocular vision, whatever the viewing distance. When saccading between targets at far distance (for example $400 \mathrm{~cm}$ ), the saccade starts with visual axes almost parallel (small vergence angle); coordination of saccade allows the angle to be maintained. At near distance (for example $40 \mathrm{~cm}$ ), the eyes converge; yet the saccades should move the eyes by the same amount in the same direction so that the initial angle of convergence is again maintained. By which mechanisms the central nervous system (CNS) succeeds in such coordination is a controversial issue since early debates between Hering (1868) and Helmholtz (1856-1866). According to Hering, binocular coordination is based on neuroanatomic connections, allowing the same innervation to be sent to conjugate muscles so that the eyes move as a single organ. According to Helmholtz, neuroanatomic connections would be separate for each eye and thus binocular coordination of movements could be reached only via training and binocular visual experience.
Hering's thinking dominated both basic and clinical research. Electrophysiological evidence in favour of Hering's law of equal innervation has been presented in monkeys: burst saccade neurons project to motoneurons innervating the lateral, external rectus of one eye and also project to interneurons, that is, the neural relay to the motoneurons innervating the contralateral medial rectus (for a review see Leigh \& Zee, 2006). For vertical saccades, Moschovakis, Scudder and Highstein (1990) provided evidence for an even more robust structural basis allowing equal innervation of vertical recti of both eyes. Furthermore, a clinical study (Kommerell, Olivier \& Theopold, 1976) showed that spontaneous adaptation to unilateral oculomotor paresis obeys Hering's law. Indeed, the two patients studied used the paretic eye to fixate, because it had better acuity than the non paretic eye. The size of the saccades became appropriate for the paretic eye while the non paretic eye made hypermetric saccades. The adaptative increase of the gain of saccade command was equal for both eyes.

In the last three decades, however, research on combined movements in direction and in depth (e.g. from close center to far and right) revealed the existence of rapid saccades that are widely unequal for the two eyes. 
The inequality allows the appropriate vergence angle for the depth to be achieved during the saccade itself (Enright, 1984). This observation stimulated controversial interpretations, including controversial electrophysiological findings. Indeed, Mays and Gamlin (1995) reported increased activity of vergence neurons during combined movements, suggesting an acceleration of vergence by the saccade. These data support earlier models of coupling of saccade and vergence generators via the omnipausers (Zee, Fitzgibbon \& Optican, 1992). On the other hand, Zhou and King (1998) reported monocular coding of saccades in the saccade generator located at the paramedian reticular formation. Based on such electrophysiological observations, King and Zhou (2002) proposed a model of control in line with Helmholtz' thinking: the site of learning of binocular coordination would be downstream at the level of oculomotor nuclei rather than at the central saccade command level.

The hypothesis of Hering's law for equal central innervation of the eyes is not incompatible with the necessity for learning. Indeed, the proprieties of the extraocular muscles may not be identical; the relay for innervating the internal medial rectus is longer than that for innervating the external lateral rectus. Learning may be necessary to adjust the central command so that the eyes move finally by the same amount.

Thus, the question is no longer whether learning is necessary for achieving fine binocular coordination of saccades, but instead, what are the mechanisms used to achieve such coordination and at which level this learning takes place. Our driving hypothesis is that the CNS uses natural existing mechanisms of saccade-vergence interaction to render saccades equal for the two eyes.

To illustrate this hypothesis, one can consider the case of horizontal rightward saccades: because of the asymmetry of the oculomotor plant (difference in muscles and/or in relays of innervation), a central saccade command would result in a saccade that was larger in the abducting than the adducting eye (Fig. 1A). We hypothesize that the CNS learns to couple the conjugate saccade command with an appropriate rapid vergence command tailored to reduce the initial inequality of the saccade (Fig. 1B). Thus the resulting saccade has only a small residual divergent disconjugacy that could be subsequently corrected by convergent post saccadic drift, as we see frequently in adults (Collewijn, Erkelens \& Steinman, 1988a; Zee et al., 1992). Convergence has to occur rapidly during the saccade; this is possible as shown by the studies on combined movements in direction and in depth (Enright, 1984). When the eyes are already converging at near distance, the CNS learns to program an additional convergence command to eliminate the peripheral asymmetry. Perhaps under such circumstances it is more difficult to tailor the size of the rapid intrasaccadic convergence needed to cancel the peripheral asymmetry.
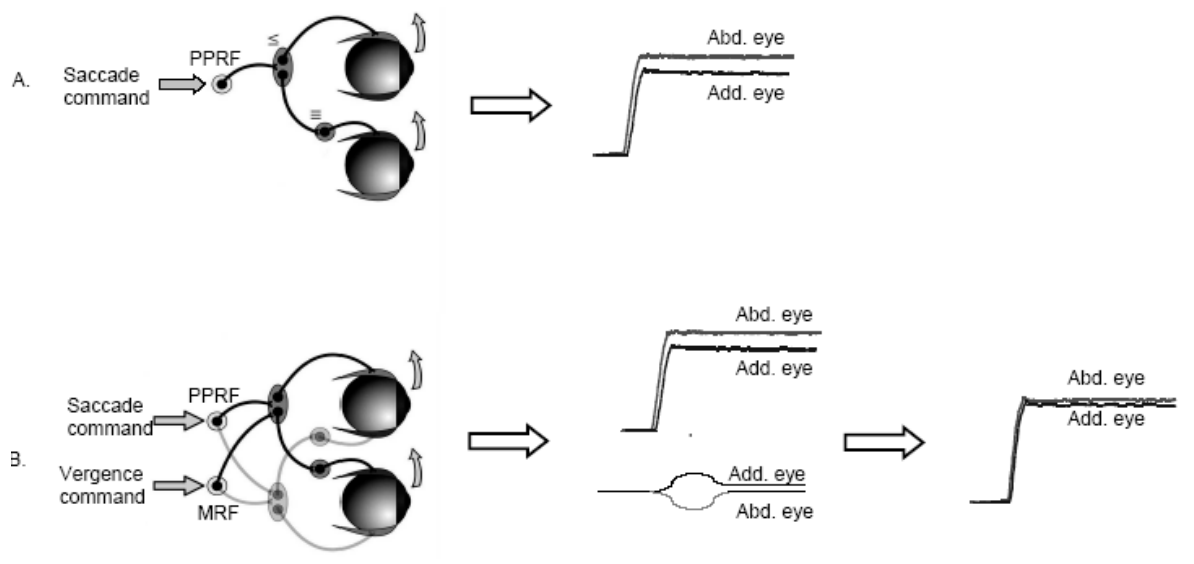

Figure1. A. Hering's view: The lateral rectus of the abducting eye and the contralateral medial rectus of the adducting eye receive an equal saccadic command. Asymmetry of viscoelastic properties of muscles and/or delay in innervation cause unequal saccades. B. Hypothesis: A small vergence command is sent together with the saccades, compensating the peripheral asymmetries. PPRF: paramedian pontine reticular formation. MRF: mesencephalic reticular formation. III: oculomotor nucleus. IV: abducens nucleus. Note that the pathway for vergence is hypothetical. 
The hypothesis is based on the idea that both the saccade and the vergence subsystems obey Hering's law (same initial innervation sent to the two eyes). In addition to using naturally existing physiological mechanisms; this hypothesis goes against a modular view of the oculomotor control. Most of the ecological eye movements change the direction and the depth of the gaze, and the CNS uses the oculomotor system in its integrity rather than distinct saccade and vergence modules.

Next we will review studies of healthy subjects and patients, supporting the above mentioned conceptual model, that is, supporting the contention that binocular coordination depends on vergence.

\section{Development of binocular coordination in children}

Fioravanti, Inchingolo, Pensiero and Spanio (1995) reported poor binocular coordination of saccades in young children, improving with age. Yang and Kapoula (2003) reinvestigated this issue comparing children aged from 4.5 to 12 years and adults aged from 22 to 45 years. They recorded binocularly, with an infrared system (Oculometer Dr. Bouis), horizontal saccades of $20^{\circ}$ at near (20 $\mathrm{cm})$ and at far distance $(150 \mathrm{~cm})$; the corresponding convergence angle was respectively $17^{\circ}$ and $2^{\circ}$.

At the far distance the quality of coordination of saccades achieved the adult level for the participants aged 78 years while at near distance the coordination remained poor until the age of 10-11 years (Fig.2). Moreover, saccade disconjugacy was found to be stereotyped in adults, mostly divergent, while in children saccade disconjugacy could be divergent or convergent at similar rates. These results clearly show that the quality of coordination of saccades is based, at least partially, on learning. Learning is complex and involves specific adjustments according to viewing distance and to the vergence angle: the data support the idea of greater difficulty in learning to produce appropriate intrasaccadic convergence when the eyes are already converging.

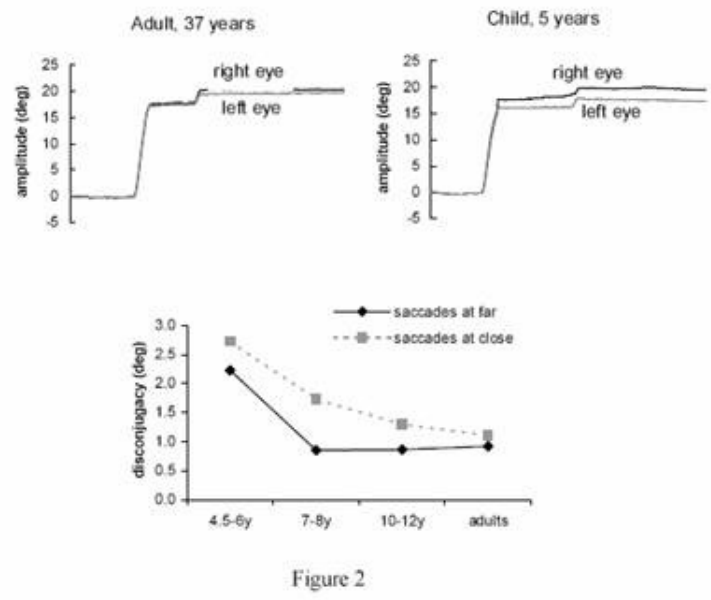

Figure 2. Up: Example of binocular recording of a rightward saccade from an adult and a child (5 years old). Bottom: Saccade disconjugacy for children as a function of their age. In the graph, adult data are shown on the right. The saccade disconjugacy in children is dramatically deteriorated at close distance, more than was the case for adults.

Poor binocular alignment during fixation in children was also reported during reading by Blythe, Liversedge, Joseph, White, Findlay and Rayner (2006). Children (711 years) and adults (18-21 years) were asked to read single sentences with a target word in the middle of each sentence. Fixation disparity was examined at the beginning and at the end of the fixation. At the beginning of the fixation the disparity was on average 1.53 character spaces for children and 1.26 character spaces for adults (a character space corresponding to $0.19^{\circ}$ ). Initial fixation disparity was predominantly divergent, followed by convergent drift of the eyes; these observations were particularly true for adults. The finding of divergent fixation disparity at the beginning of fixation are in line with those reported previously by Fioravanti et al. (1995) and Yang and Kapoula (2003) on binocular coordination of saccades.

Thus binocular coordination of saccades and binocular eye alignment during fixation is learned during childhood. Next we will present studies showing that such learning relies on the quality of vergence state. 


\section{Vertigo, migraine and vergence abnormalities in children}

Anoh-Tanon, Bremond-Gignac and Wiener-Vacher (2000) reported that a substantial number of children (more than 5\% consulting the ENT - Ear Nose Throat service for vertigo, headaches and equilibrium disorders) showed normal vestibular function but revealed vergence abnormalities in clinical orthoptic tests. Several subsequent studies from our team objectivized these clinical observations with vergence and saccade eye movement recordings. Bucci, Kapoula, Yang, Wiener-Vacher and Bremond-Gignac (2004b) conducted eye movement studies for 12 of these children presenting vertigo without measured vestibular abnormalities. Oculomotor findings were: normal latency for saccades but abnormally slow latency for vergence, alone or combined with saccades; slowness of initiation was particularly pronounced for convergence. In the same study, the authors examined the effect of the orthoptic vergence training: after 12 sessions of vergence exercises, latency was reduced even though that of convergence remained above normal levels. In a subsequent study, Bucci, Kapoula, Yang, BremondGignac and Wiener-Vacher (2004a) reported abnormalities in the accuracy and in the speed of vergence and of combined saccade-vergence movements. Such deficits, particularly the speed abnormality, were attributed to downstream brainstem dysfunction, perhaps poor interaction between the saccade and the vergence generators. Again, orthoptic vergence training improved the accuracy of vergence movements even though post-training performance remained under normal levels. Finally, Bucci, Kapoula, Bremond-Gignac and Wiener-Vacher (2006) examined the binocular coordination of saccades at far and at near distances in 15 children with vertigo. At far distance, the binocular coordination of saccades was normal, that is, a small residual disconjugacy was observed ( $\leq 5 \%$ of the amplitude of the saccade), similar to that found in normal adults. In contrast, for saccades at near distance (with the eyes converging by $17^{\circ}$ ), coordination was poor (about $10 \%$ of saccade amplitude). Examples of saccades from one of these children is seen in
Fig. 3. The drift of the eyes after the saccade is also abnormally increased for saccades at near distance. After 12 sessions of orthoptic training of vergence, the disconjugacy of the saccades dropped to normal values for the near distance, that is, to about $5 \%$ of the saccade size. Improvement for the binocular coordination of the saccades was systematic while the improvement of the disconjugate post saccadic drift did not reach statistical significance. This study provides clear evidence for the hypothesized link between the vergence and the binocular coordination of the saccades, namely the idea that adaptive mechanisms needed to develop normal binocular coordination of saccades would be based on continuous interaction between the saccade and the vergence systems. The observations in children with vertigo are in line with prior studies in adult patients with trochlear nerve paresis: Lewis, Zee, Repka, Guyton and Miller (1995) observed improvement of binocular coordination in vertical saccades after surgery, that was positively correlated with their pre-surgical capabilities for vertical vergence.

In the child studies (Bucci et al., 2006; Bucci et al., 2004a; Bucci et al., 2004b), slow initiation of vergence, low accuracy, slow speed, absence of acceleration of vergence by the saccade would also imply a difficulty in tailoring the hypothetical rapid convergence command aiming to render the saccade more equal for the two eyes (i.e. reducing the peripheral asymmetries). Orthoptic training, perhaps acting via attentional and motor learning mechanisms, would improve vergence capacity: even though not very specific, this type of training can still be useful for improving vergence performance, consequently improving the binocular coordination of saccades. Thus, the vergence subsystem could act as a calibrator of binocular saccadic control. The importance of vergence as a calibrator of other oculomotor subsystems is known. For example, for the vestibular system, the gain of vestibuloocular function increases with decreasing viewing distance. Vergence and related afferent and efferent signals are probably used for this adjustment (for a review see Leigh \& Zee, 2006). 
Saccades at far

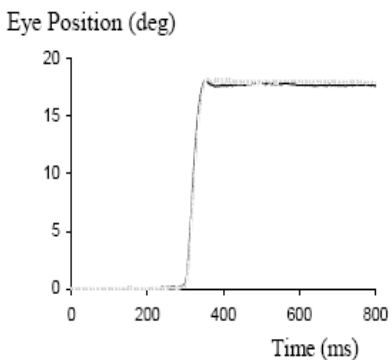

before

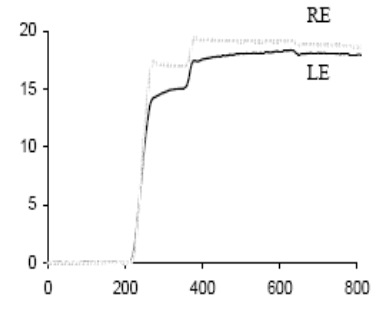

Saccades at near

after orthoptic training

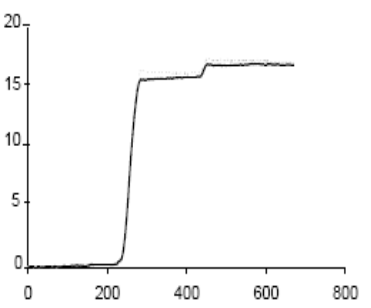

Figure 3. Binocular recordings of saccades from a child with vertigo recorded at far and at near distance before and after orthoptic training. The grey trace is the right eye, the dark trace is the left eye; target-LED appeared at time zero.

\section{Dyslexia and vergence problems}

Dyslexia is a complex functional problem and it is still controversial whether oculomotor deficits are more frequent in dyslexic children than in non-dyslexic children.

Stein, Riddell and Fowler $(1987 ; 1988)$ reported that fixation instability and reduced vergence capacity occurred frequently in dyslexics; yet they did not quantify the range of convergence and divergence capacities in such children. Evans and Drasdo (1990) reported similar observations, e.g. significantly lower convergence and divergence capacities relative to non-dyslexics; but did not find a correlation between visual deficits and reading disability. Ygge Lennerstrand, Ryberg, Wijecoon and Petterson (1993) examined 86 dyslexics and 86 nondyslexics; they reported that problems of accommodation, stereo-acuity, vergence or strabismus occurred at similar rates in dyslexics and non-dyslexics. However in another study the same group reported visual deficits in dyslexics, such as lower contrast sensitivity (Ygge, Lennerstrand, Axelsson \& Rydberg, 1993).

Kapoula, Bucci, Jurion, Ayoun, Afkhami and Bremond-Gignac (2007) performed exhaustive orthoptic evaluation in 57 dyslexics and 47 non-dyslexic children of comparable age (mean age: 11 years). Dyslexics and non-dyslexics were both recruited mostly from a college in Paris with classes specialized for dyslexia. Dyslexics were diagnosed before entering the college by a specialized medical centre, using standard criteria. Examination included neurological/psychological and phonological tests, repeated before our oculomotor test. The speed of reading, text comprehension, and the capacity of reading word/pseudowords was also evaluated by using the L2MA battery (Chevrie-Muller, Simon \& Fournier, 1997). All children included in the study had reading age more than two SD behind that expected from their age. The intelligence quotient (IQ) was evaluated with WISC III, and was in the normal range, i.e. between 85 and 115 . Non-dyslexic children were recruited predominately from the same college and they had normal school performances, most of them superior to the mean scores of their class.

Table 1 Percentages of dyslexic and non-dyslexic children with stereoacuity (measured with the TNO test) better or equal to 60 seconds of arc and percentages of children with near point of convergence $(N P C)$ below $6 \mathrm{~cm}$

\begin{tabular}{|c|c|c|c|}
\hline \multirow[t]{2}{*}{ Subjects } & \multicolumn{2}{|c|}{ TNO (sec. of arc) } & \multirow{2}{*}{$\begin{array}{c}\mathrm{NPC}(\mathrm{cm}) \\
\leq 6\end{array}$} \\
\hline & $<60$ & 60 & \\
\hline Dyslexics (57) & $21 \%$ & $78 \%$ & $58 \%$ \\
\hline Non dyslexics (46) & $31 \%$ & $69 \%$ & $85 \%$ \\
\hline
\end{tabular}

Orthoptic examination was conducted by orthopticians or optometrists. The major orthoptic findings were: normal scores in the TNO stereoacuity test (60 seconds of arc) were slightly more frequent in dyslexics than in nondyslexics; yet, stereoacuity scores better than normal $(<$ 60 seconds of arc) were slightly more frequent in nondyslexics (Table 1). In contrast, normal values of the near 
point of convergence (NPC), that is, below $6 \mathrm{~cm}$, occurred in $72 \%$ of non-dyslexics while only $44 \%$ of dyslexic children reached this value. Many dyslexics (42\%) showed NPC superior to $7 \mathrm{~cm}, 6 \%$ of them beyond 10 $\mathrm{cm}$. Divergence amplitude was measured with the bar of prism that examines the disparity driven divergence. This test was carried out both at near $(40 \mathrm{~cm})$ and at far distances $(400 \mathrm{~cm})$. It should be noted that at near distance divergence response to the prism could be partially assisted by a mechanism of relaxation of convergence, while such a mechanism cannot be involved when the child fixates at far distance. Divergence amplitudes elicited by the prism were lower both at near and at far distance: $10 \mathrm{pD}$ versus $12 \mathrm{pD}$ at near distance and $4 \mathrm{pD}$ versus $6 \mathrm{pD}$ at far distance, for dyslexics and non-dyslexics respectively (Fig. 4). As shown in Fig. 4, low values of divergence are more frequent in dyslexics than in nondyslexics and reciprocally high values of divergence are more frequent in non-dyslexics than in dyslexics.

Kapoula et al. (2007) argue that reduced divergence capacity at far distance $(400 \mathrm{~cm})$ indicates the presence of a deficit of divergence per se, occurring independently from convergence and accommodation. Indeed, physiological studies indicate distinct control of convergence and divergence both at the cortical and subcortical levels. At the cortical level Gamlin and Yoon (2000) identified an area close to the frontal eye fields containing different subgroups of cells that discharge before and during convergence and divergence movements respectively. An EEG study from Tzelepi, Lutz and Kapoula (2004) indicated that the cortical activation prior to divergence movements was more posteriorly confined than that prior to convergence. At the subcortical level, Mays (1984) identified in the monkey mesencephalic reticular formation distinct groups of cells activated prior and during divergence versus convergence eye movements. Kapoula et al. (2007) concluded that specific vergence abnormalities assessed by orthoptics occur more frequently among dyslexics. This observation motivated studies on the binocular coordination of saccades in dyslexics (see below).

Divergence fusion capacity (prism D)
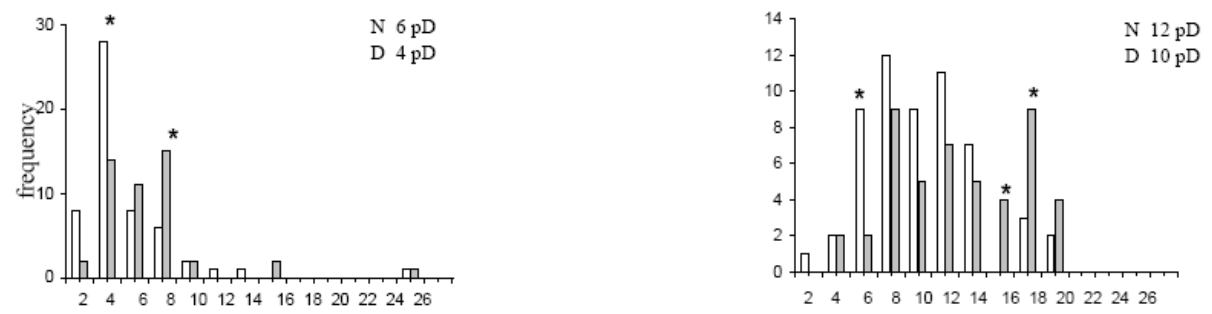

$\square$ Dyslexics

$\square$ Normals

Figure 4. Divergence fusion capacity distribution for dyslexic (white bars) and normal (grey bars) children at far and near distance. The median of dyslexics and normals is also given. Asterisks indicate a significant difference between dyslexics and normals.

\section{Binocular coordination of saccades in dyslexic children}

Bucci, Bremond-Gignac and Kapoula (2007) studied the binocular coordination of saccades in 18 dyslexic children (average age 11.4 years) compared with 13 non- dyslexic children of comparable age (11.2 years). They used a simplified single word reading task (introduced by F.Vitu). The task was carried out at a near viewing distance $(40 \mathrm{~cm})$ on a computer screen. Briefly each trial started with the presentation of the sign ' + ' on the left 
side of the screen. After $500 \mathrm{~ms}$ the ' + ' was replaced by a cross and simultaneously a word was presented in the middle of the screen and a ' + ' on the right side. The child was invited first to fixate the cross on the left side, then to read the word silently and then to fixate the ' + ' on the right side. Saccades from this task were compared with another standard saccade task during which the child had to fixate an LED that jumped randomly from $0^{\circ}$ to $10^{\circ}$ or $20^{\circ}$ to the right or to the left; remaining at each location for $2 \mathrm{~s}$.
The amplitudes of the saccades during the word reading task ranged between $6.5^{\circ}$ and $10^{\circ}$, depending on the length of the word and were similar for the two groups of children. The amplitudes of the saccades during the LED task were about $15^{\circ}$ and were similar for dyslexics and non-dyslexics. Thus the binocular coordination of saccades presented next concerns similar saccade amplitudes for dyslexics and non-dyslexics.

Dyslexic child Non-dyslexic child

Left and Right eye position
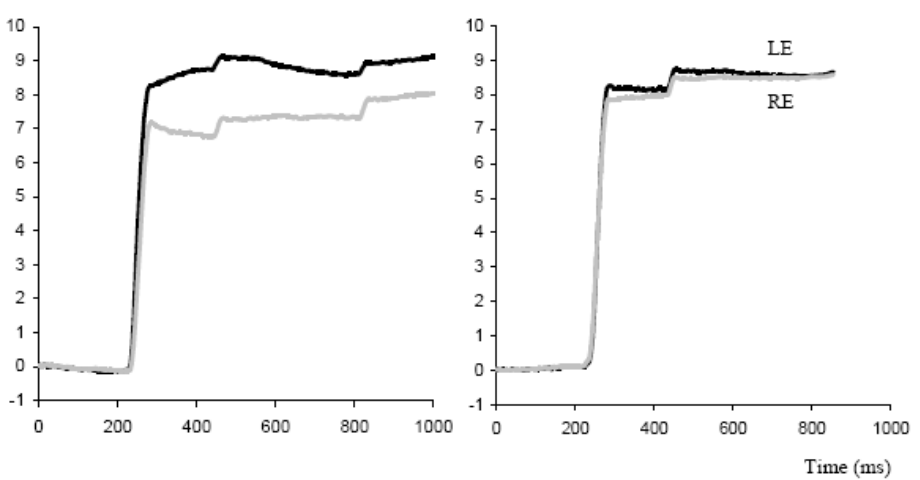

Figure 5. Binocular recordings of saccade during reading a word from a dyslexic and a non-dyslexic child. Individual eye positions are shown for the left eye (dark trace) and the right eye (grey trace); the word appeared at time zero.

Fig. 5 shows typical binocular recording of saccades from a dyslexic child and a non-dyslexic child in the word reading task. Saccades are poorly coordinated for the dyslexic child and are followed by large disconjugate drift. Quantitative results from this study showed a statistically significant difference in the disconjugacy of the saccades between the two groups of children: for dyslexics the mean disconjugacy of saccades was $1.17 \pm 0.11^{\circ}$ and $0.95 \pm 0.07^{\circ}$ respectively for the word reading task and the LED-target task. In contrast, for non-dyslexics the mean disconjugacy was only $0.41 \pm 0.05^{\circ}$ for saccades to words, and $0.34 \pm 0.02^{\circ}$ for saccades to LED targets (Fig. 6A). Another important observation from this study concerned the disconjugate post-saccadic drift measured in the first period of the fixation until the corrective saccade (Fig. 6B). The mean disconjugate drift was significantly larger in dyslexics $\left(0.45 \pm 0.05^{\circ}\right.$ and $0.46 \pm 0.07^{\circ}$, respectively for the word reading task and for the LED-target task). Non-dyslexics showed smaller values $\left(0.25 \pm 0.03^{\circ}\right.$ and $0.26 \pm 0.03^{\circ}$ respectively for the word reading task and the LED-target task). 


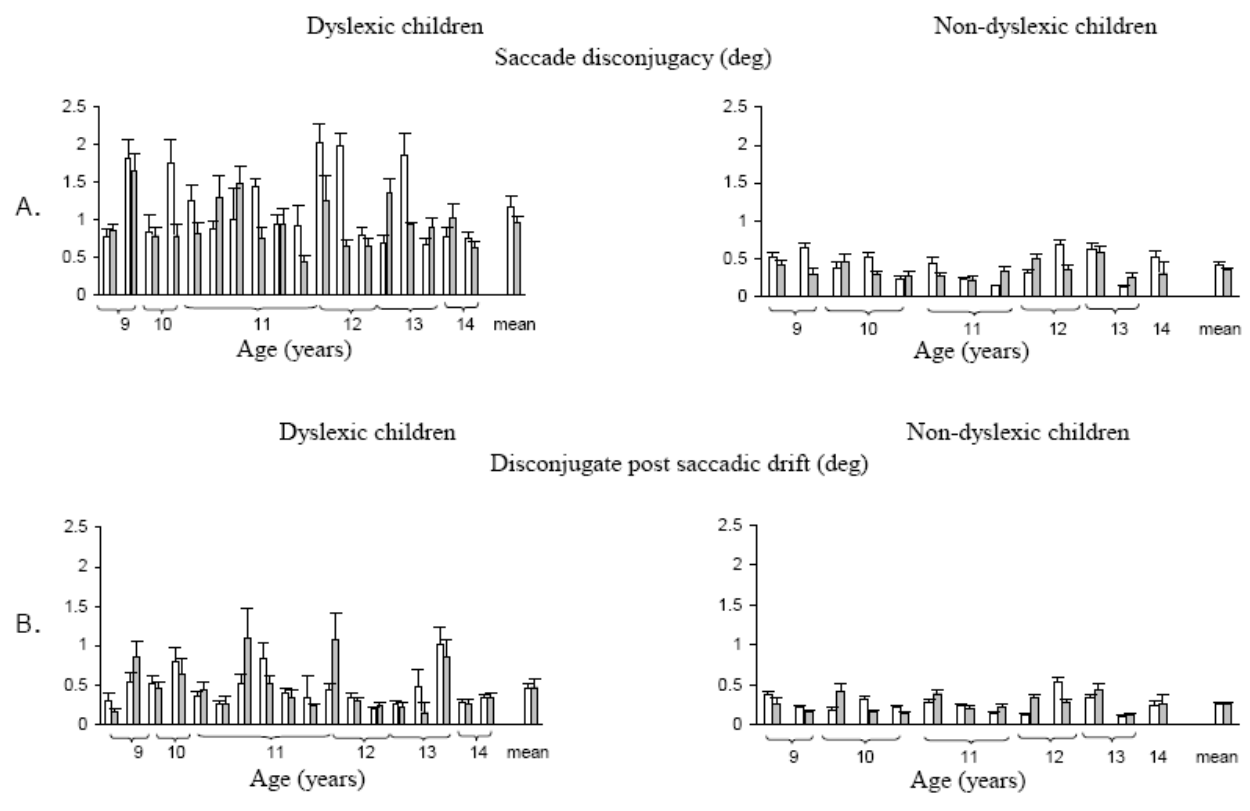

Figure 6. Individual average disconjugacy of saccades in degrees (A) and individual average disconjugate post saccadic drift in degrees $(B)$ measured during reading of single words and during saccades to the LED for dyslexic and non-dyslexic children.

Another important observation concerns the lack of correlation between the saccade disconjugacy and the post-saccadic drift disconjugacy in dyslexics (Fig. 7). For non-dyslexics the majority of saccades had divergent disconjugacy while the disconjugacy of post saccadic drift was predominantly convergent (see positives values on the y axis); the correlation was statistically significant $(\mathrm{r}=0.35, \mathrm{p}<0.05)$. In contrast, for dyslexics, the disconjugacy was almost randomly convergent or divergent; similarly the disconjugacy of the post saccadic drift was either convergent or divergent at similar rates and there was no correlation between the disconjugacy and the post saccadic drift.

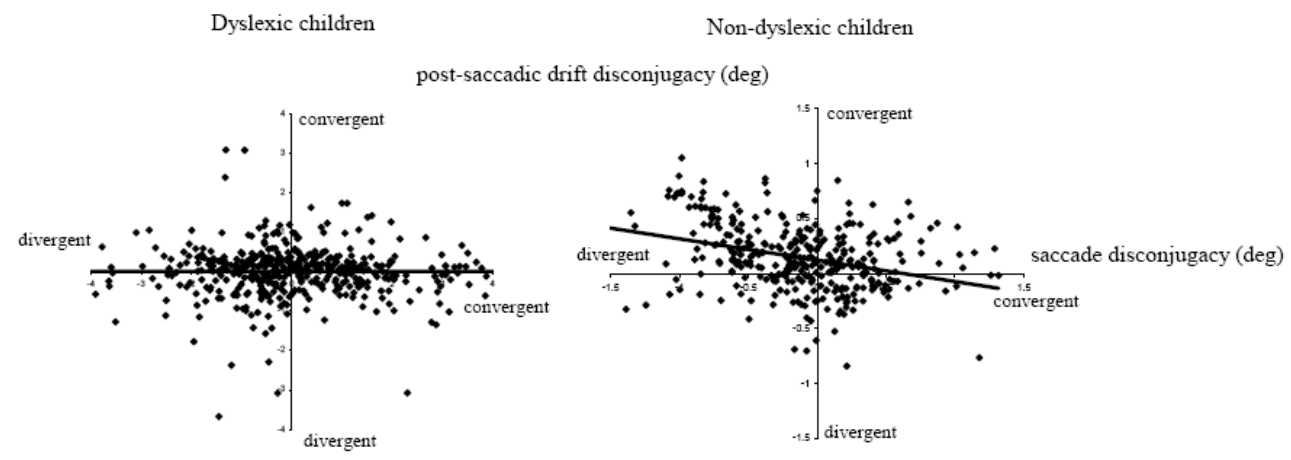

Figure 7. Relationship between saccade and drift disconjugacy. For each saccade in the reading task condition, the disconjugacy of the saccade is shown in degrees ( $X$-axis) and the disconjugacy of the post-saccadic drift ( $Y$-axis). Positive values indicate convergent disconjugacy, negative values divergent disconjugacy. Note the different scale for both diagrams. 
To our knowledge, this study was the first to quantify the quality of binocular coordination during and after saccades in dyslexic participants, showing that the coordination is poor in both tasks whether it be reading single words or making saccades to LED targets. Recall that both tasks were run at near distance. Whether this deficit in dyslexics is specific to the near distance is not yet known. The lack of the stereotypical pattern, that is, divergent intrasaccadic disconjugacy followed by convergent disconjugate post saccadic drift implies that dyslexics are exposed to substantial binocular disparity during the fixation period following the saccade (as the disconjugate post-saccadic drift does not systematically reduce the disparity resulting from the intrasaccadic disconjugacy). Whether such disparity interferes with visual processing of words needs further investigation. Dyslexics may have to force their sensory fusional system to obtain single vision of the word. Continuous sensory effort during reading can cause visual fatigue. Further investigation with text reading should combine physiological measures of binocular coordination of saccades, of residual fixation disparity and measure of reading performance and of visual fatigue.

To summarize, studies of our group in young normal children, in older children with vertigo symptoms or with dyslexia clearly show a difficulty in coordinating saccades for the two eyes at near distance, when the eyes are converged. Moreover, children with vertigo symptoms and dyslexic children both show during the orthoptic clinical examination vergence abnormalities (remote NPC and reduced divergence capacities). These observations also support the hypothesis of a link between vergence and binocular coordination.

\section{Binocular coordination of saccades during free exploration of paintings in dyslexics and non-dyslexics}

For all above cited studies, binocular coordination of saccades was examined during laboratory tasks involving repetitive saccades in the horizontal direction. Indeed, during reading, most of the saccades are horizontal (from left to right) and are part of a well learned motor sequence. It is unknown whether problems of binocular coordination of saccades in dyslexics exist in free exploration tasks. Kapoula, Bucci, Ganem, Poncet, Daunys and Bremond-Gignac (in press) examined the binocular coordination of saccades in dyslexics and non-dyslexics during free exploration of cubist artwork from Fernand Léger.

The study was conducted in 15 dyslexic children (mean age 11.2 years) and 8 non-dyslexics of comparable age (mean 11.1 years). Children were seated in front of a computer screen placed at $30 \mathrm{~cm}$. Initially, they fixated a line target on the bottom-left of the screen; then, the image of one of three paintings was displayed on the screen, for a period of 30 seconds. The angular size of the painting on the screen was $18 \times 25.4^{\circ}$. Children explored spontaneously the painting and no specific instructions were given. In a control condition, children performed visually-guided saccades. The targets were nonius lines (two vertical segments of $0.9^{\circ}$, each misaligned horizontally by $\left.0.1^{\circ}\right)$. The nonius lines jumped from the center of the screen to the left or to the right ( $7^{\circ}$ of eccentricity) and then returned to the center of the screen. From the eye movements during the free exploration were extracted pure horizontal saccades, e.g. saccades where the eyes were moving almost exclusively in the horizontal plane with a deviation in the vertical direction $\leq 1^{\circ}$. The rationale was to assess if problems of horizontal binocular coordination, previously studied for simple tasks involving repetitive saccades or for reading, would also exist during spontaneous and free exploration. Horizontal saccades and fixations during free exploration were compared with the horizontal saccades and fixations in the control condition. 

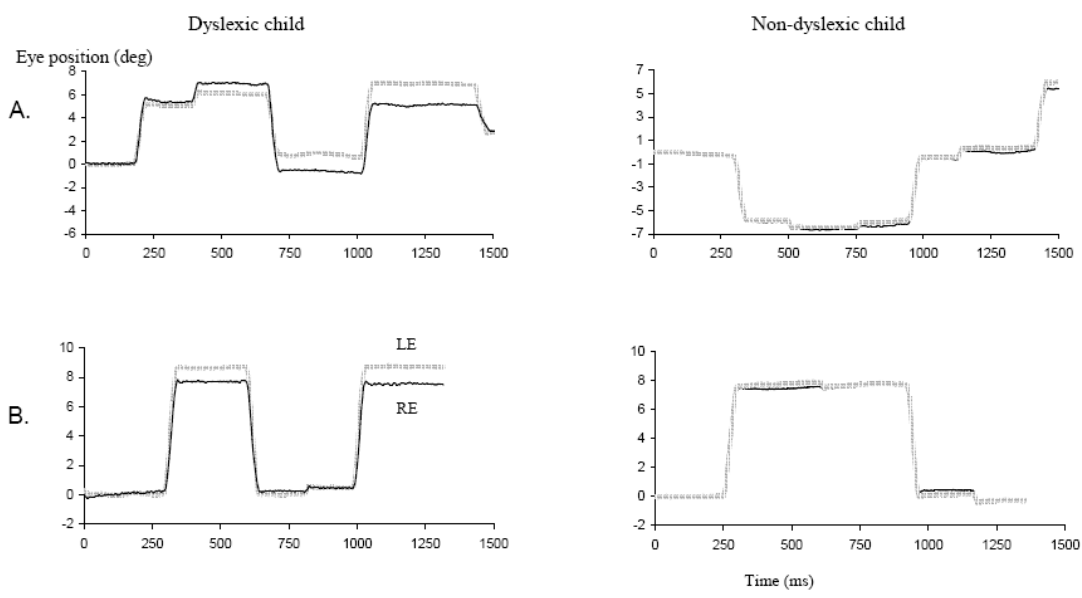

Figure 8. Typical binocular recordings of horizontal saccades during painting exploration (A) and during fixating nonius lines (B) from a dyslexic and a non-dyslexic child. For the dyslexic, the saccade is larger in the right eye, so that the eyes are not aligned at the end of the saccade but they cross or uncross relative to their position at the beginning of the saccade. During the fixation period following the saccade the eyes are drifting mildly but the drift can further increase the misalignment of the eyes due to the saccade. For the non-dyslexic child the disconjugate drift of the eyes reduces the crossing or uncrossing of the eyes caused by the saccade.

The mean size of exploration saccades was similar $\left(5.2^{\circ} \pm 0.6^{\circ}\right.$ for dyslexic and $5.1^{\circ} \pm 0.9^{\circ}$ for nondyslexic). Fig. 8 shows a sequence of horizontal saccades during free exploration (Fig. 8A) and during the control condition (Fig. 8B) from a dyslexic and a non-dyslexic child. Clearly, the dyslexic child shows larger disconjugacy than the non-dyslexic child in the free exploration $\left(0.7^{\circ}\right.$ versus $\left.0.2^{\circ}\right)$ and in the nonius-line condition $\left(0.5^{\circ}\right.$ versus $0.18^{\circ}$ ). The ANOVA applied on the disconjugacy measure showed a significant group effect $(\mathrm{F}(1,28)=$ 251.05, $\mathrm{p}<0.001)$, a significant effect of experimental condition $(\mathrm{F}(1,28)=33.46, \mathrm{p}<0.001)$, and a significant interaction between group and experimental condition $(\mathrm{F}(1,28)=15.07, \mathrm{p}<0.005)$. This indicates that the difference between free exploration and single target measures was greater in dyslexics than in non-dyslexics. A post-hoc test (LSD) for dyslexics was significant ( $p<$ 0.001 ), while for non-dyslexics the difference was not significant.

Disconjugate post-saccadic drift during free exploration was significantly higher relative to the control condi- tion, for both non-dyslexic $\left(0.33^{\circ}\right.$ versus $\left.0.23^{\circ}\right)$ and dyslexic children $\left(0.34^{\circ}\right.$ versus $\left.0.23^{\circ}\right)$.

For non-dyslexics, most of the saccades had divergent disconjugacy $(68 \%)$ while the drift that followed was mostly convergent $(90 \%)$. The correlation between the two was significant (coefficient of correlation of Bravais Person $r=0.42, p<0.03$, see Fig. 9). In contrast, for dyslexics, $46 \%$ of the saccades showed divergent disconjugacy and $51 \%$ of the post-saccadic drifts were convergent; the amplitude of the disconjugacy of the saccades was larger and variable and there was no significant correlation between saccade disconjugacy and post-saccadic disconjugate drift $(r=0.02)$. Thus, even during free exploration, non-dyslexics produced a stereotypically correlated pattern in which disconjugate post saccadic drift reduced the disconjugacy that occurred during the saccade while in dyslexics the two phenomena were again uncorrelated. Our new study shows that saccade disconjugacy in dyslexics can occur independent of reading processes; it seems to be an intrinsic physiological problem, leading to loose control of binocular eye alignment. 


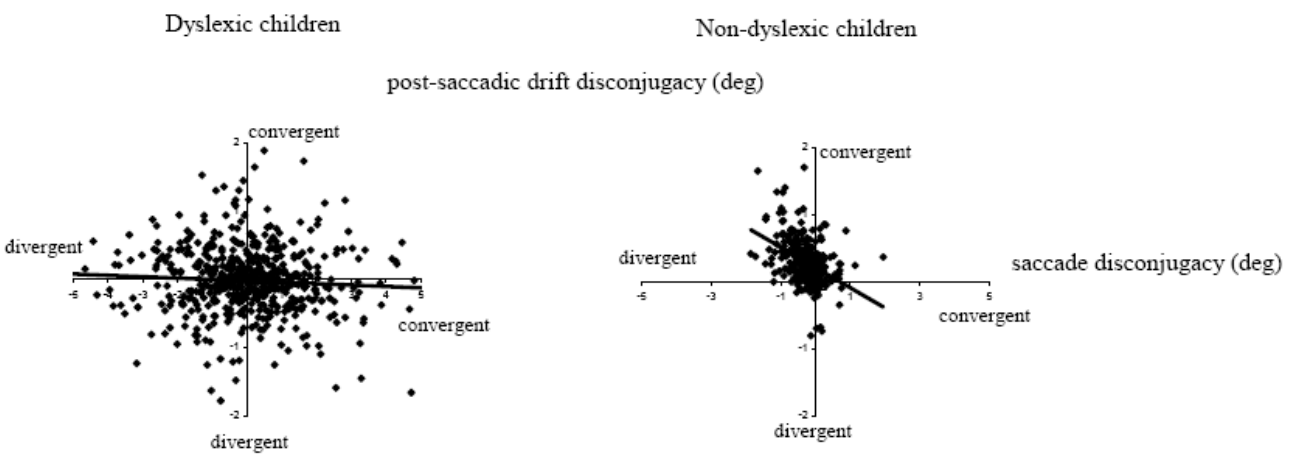

Figure 9. Relationship between saccade and drift disconjugacy. For each saccade in the free exploration condition, the disconjugacy of the saccade (X-axis) and the disconjugacy of the post-saccadic drift (Y-axis) is shown in degrees. Positive values indicate convergent disconjugacy, negative values divergent disconjugacy.

Convergent or divergent post saccadic drift during the fixation period could be either beneficial or destructive for obtaining binocular vision, depending on the disconjugacy of the preceding saccade, and thus, on the residual disparity remaining at the end of the saccade; uncorrelated intra / post-saccadic behavior occurs in dyslexics only. We believe that physiological mechanisms of binocular coordination can be better understood when examining both saccades and fixation drift. Studies isolating binocular coordination during the fixation (Blythe et al., 2006; Liversedge, White, Findlay \& Rayner, 2006b) give only a limited view of the physiological mechanism involved.

Poor binocular control in dyslexics could reflect immaturity of saccade-vergence interaction learning mechanisms, needed to coordinate saccades, particularly at near distances (i.e. with the eyes converging). One of the theories about the origin of the dyslexia supposes a deficit in the magnocellular system, the posterior parietal cortex being a major site of this system (for example, Livingstone, Rosen, Drislane \& Galaburda, 1991; Stein \& Walsh, 1997). As will be presented next, the posterior parietal cortex is actively involved in the binocular coordination of saccades and perhaps keeps the brainstem and cerebellar circuits calibrated. Thus, poor binocular coordination in dyslexics could be one of the multiple manifestations of the immaturity of the circuits involving the magnocellular system, the cerebellum and the brainstem. It is probably not the cause of dyslexia but it could be an aggravating factor, particularly during reading acquisition. An ongoing longitudinal study tests the persistence of poor binocular coordination in dyslexics up to 17 years and its relation to reading performance. It is common experience that reading is possible even with low visual quality input; however such reading over long period of time becomes tiring. Juhasz, Liversedge, White and Rayner (2006) and Liversedge, Rayner, White, Findlay and McSorley (2006a) reported increased fixations duration with case alternation or dichoptic presentation of the target word. Perhaps such increase could be indicative of fatigue.

Problems of binocular coordination of saccades seem to be similar in children with dyslexia and children with vertigo symptoms; in both cases, clinically assessed abnormalities of vergence are frequent. This is probably related to the fact that learning of binocular coordination relies on a fragile system, the vergence system, dysfunctioning for both populations. Studies of vertigo and headache symptoms in dyslexics are, to our knowledge, lacking; similarly, assessment of frequency of dyslexia in children with vertigo symptoms remains to be investigated. Such comparative studies could give new insight into the role of basic physiological binocular visuomotor maturation in reading capacity.

The problems of binocular coordination, frequently associated with vergence deficits, bring new insight on the Hering and Helmholtz controversy. Indeed, in modern 
conception of the Helmholtz position (King \& Zhou, 2002), combined eye movements in direction and depth and a fortiori in direction only - are achieved predominantly via the activation of monocular saccade generators. No obvious link between vergence deficits and binocular coordination of saccades is expected in this case. Alternatively, in the modern conception of the Hering position (Busettini \& Mays, 2005; Zee et al., 1992), both saccade and vergence sub-systems are activated during combined movements in direction and depth. We go further along this line and propose permanent co-activation of saccade and vergence sub-systems, even when saccading at a fronto-parallel plane. This reasoning explains the link between vergence deficits and poor binocular coordination of saccades.

\section{Binocular coordination of vertical saccades in adults and in elderly}

Binocular coordination of vertical saccades, unlike that of horizontal saccades, has been little investigated. Collewijn, Erkelens and Steinman (1988 a, b) reported that in normal humans (four subjects aged 35, 36, 51 and 60 years), vertical yoking of vertical saccades was almost perfect as the difference between amplitudes of saccades made by left and right eye was not statistically significant: vertical disonjugacy (i.e. the difference between the two eyes) during vertical saccades was much smaller (about $0.2^{\circ}$ for vertical saccades of $20^{\circ}$ of amplitude) relative to horizontal disconjugacy for horizontal saccades of the same amplitude (about $0.6^{\circ}-1^{\circ}$ ). In contrast, the authors reported the presence of substantial horizontal vergence during vertical saccades: divergence of the eyes associated with upward saccades and convergence with downward saccades. Subsequent studies (for example, Kapoula, Eggert \& Bucci, 1996) confirmed many of these observations in a group of young adults (19-35 years).

Yang and Kapoula (2006) studied the quality of binocular control of vertical saccades in healthy subjects, 11 young adults (20-28 years) and 11 elderly adults (63-75 years). They used LED targets at $7.5^{\circ}$ or $15^{\circ}$ from the centre (fixation), up or down at two distances - near (40 $\mathrm{cm})$ or far $(150 \mathrm{~cm})$; the corresponding vergence angle was $9^{\circ}$ or $2^{\circ}$ respectively. Vertical eye movements were recorded with video-oculography (CHRONOS). The results showed that aged participants performed vertical saccades as accurately as young participants (Fig. 10). Importantly, the binocular coordination of vertical saccades was well preserved in the elderly; the mean difference between the two eyes during vertical saccades was $0.10^{\circ}$ and $0.09^{\circ}$ in young and elderly participants, respectively. Upward saccades were associated with divergence, downward ones with convergence. This secondary phenomenon was also the same direction and of similar amplitude for young $\left(1.30^{\circ}\right)$ and elderly $\left(1.25^{\circ}\right)$ participants. 
young $(22 y)$
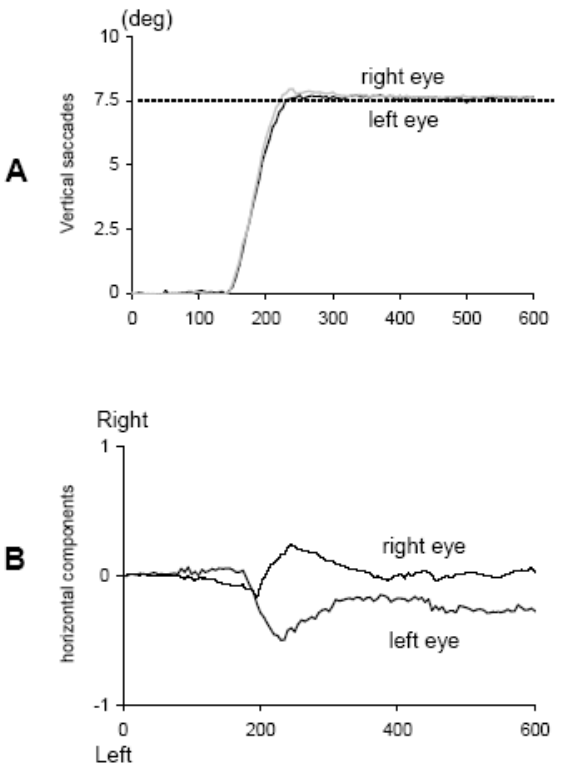

old (73y)
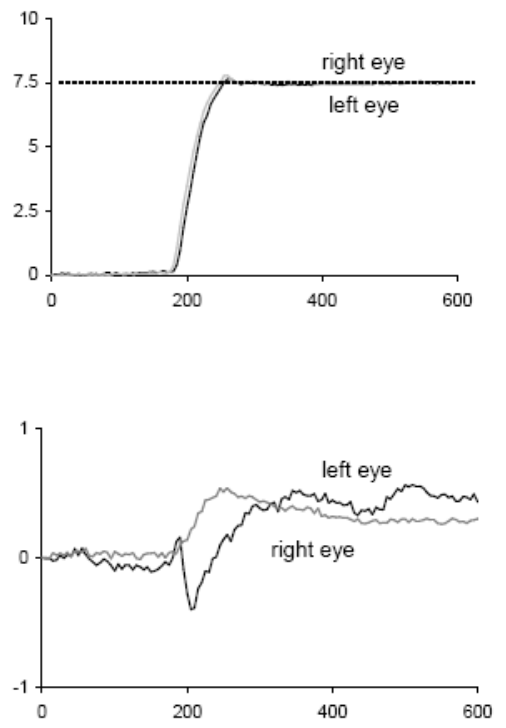

Figure 10. Examples of upward saccades from one young and one elderly participant: (A) vertical saccades for left eye and right eye, horizontal dotted line indicate target position; $(B)$ horizontal components during upward saccades.

Preservation of good accuracy and good binocular coordination of the vertical saccades in the elderly could be related to the robust control of brainstem and cerebellum of these parameters. Indeed, the rostral interstitial nucleus of the medial longitudinal fasciculus (riMLF), the key premotor substrate in the vertical gaze pathway, shows no statistically significant difference in neuronal density, glial cell density, or neuron-to-glial cell ratios between the young ( $<50$ years) and the aged $(>65$ years, see Henson, Staunton \& Brett, 2003). Magnetic resonance imaging (MRI) showed no significant ageassociated decrease in brainstem and cerebellum vermis areas in humans between 13 and 77 years (Murshed, Ziylan, Seker, Ciekcibasi \& Acikgozoglu, 2003). Extraocular muscles are also known to be unchanged over age (19-70 years, Tian, Nishida, Isberg \& Lennerstrand, 2000). The data from the elderly group are consistent with the idea of the existence of non-aging system function in the human CNS.

It remains to be seen whether binocular coordination of horizontal saccades is as well preserved in the elderly. Binocular coordination of horizontal saccades relies on adaptation, perhaps even more than that of vertical saccades. Studies in young adults have shown that properties of horizontal versus vertical adaptation are different (Bucci, Paris \& Kapoula, 2003; Eggert \& Kapoula, 1995; Kapoula, Eggert \& Bucci, 1995; van der Steen \& Bruno, 1995; Ygge \& Zee, 1995). For instance, Kapoula et al. (1995) presented images of different sizes to each eye; saccades became larger for the eye viewing the larger image and this disconjugacy persisted even under monocular viewing, indicating learning. Learning was found to be weaker for vertical saccades but less variable. Further studies are needed to test the quality of binocular coordination of horizontal saccades with ageing and on the other hand, the quality of binocular coordination of vertical saccades in children (dyslexics versus non-dyslexics). Adaptation studies in children and the elderly are completely missing. 


\section{Cerebral basis of binocular coordination of saccades}

The cerebellum is largely involved in learning and oculomotor adaptation in general (Robinson, Fuchs \& Noto, 2002; Straube, Deubel, Ditterich \& Eggert, 2001). Studies in monkeys (Takagi, Tamargo \& Zee, 2003) and in patients (Versino, Hurko \& Zee, 1996) with cerebellar lesions showed disorders in both static and dynamic eye alignment, in particular poor binocular coordination of saccades and large post-saccadic drift. Versino et al. (1996) highlighted that the flocculus and paraflocculus are important sites for the yoking of saccades and the control of both conjugate and disconjugate post-saccadic drift. The cerebellum would control eye movements through both immediate online control and long term mechanisms that keep the brainstem circuit calibrated for optimal oculomotor behavior: in patients with cerebellar lesions, the quality of binocular coordination of saccades gradually deteriorates. Other cerebral structures may be important for the coordination of saccades, in particular, the posterior parietal cortex (PPC), known to be involved in oculomotor control and visual attention in 3D space (Gnadt \& Mays, 1995).

Transcranial magnetic stimulation (TMS) is an interference method, very helpful to explore the involvement of a cerebral area in the preparation or execution of an eye movement (Elkington, Kerr \& Stein, 1992; Priori, Bertolasi, Rothwell, Day \& Marsden, 1993). Several studies using TMS have explored the role of the PPC on the initiation of saccades and vergence (Kapoula, Isotalo, Muri, Bucci \& Rivaud-Pechoux, 2001; Kapoula, Yang, Coubard, Daunys \& Orssaud, 2004; Kapoula, Yang, Coubard, Daunys \& Orssaud, 2005; Tzelepi, Yang \& Kapoula, 2005; Yang \& Kapoula, 2004). Elkington et al. (1992) showed that the TMS of the PPC increased the divergence of the eyes before the saccade, indicating that the PPC is involved in the static vergence control before eye movements. However, the study was limited to two subjects, and binocular coordination during saccades was not examined. 


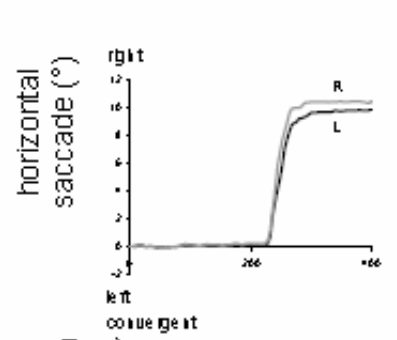

\section{Rightw ard saccades}
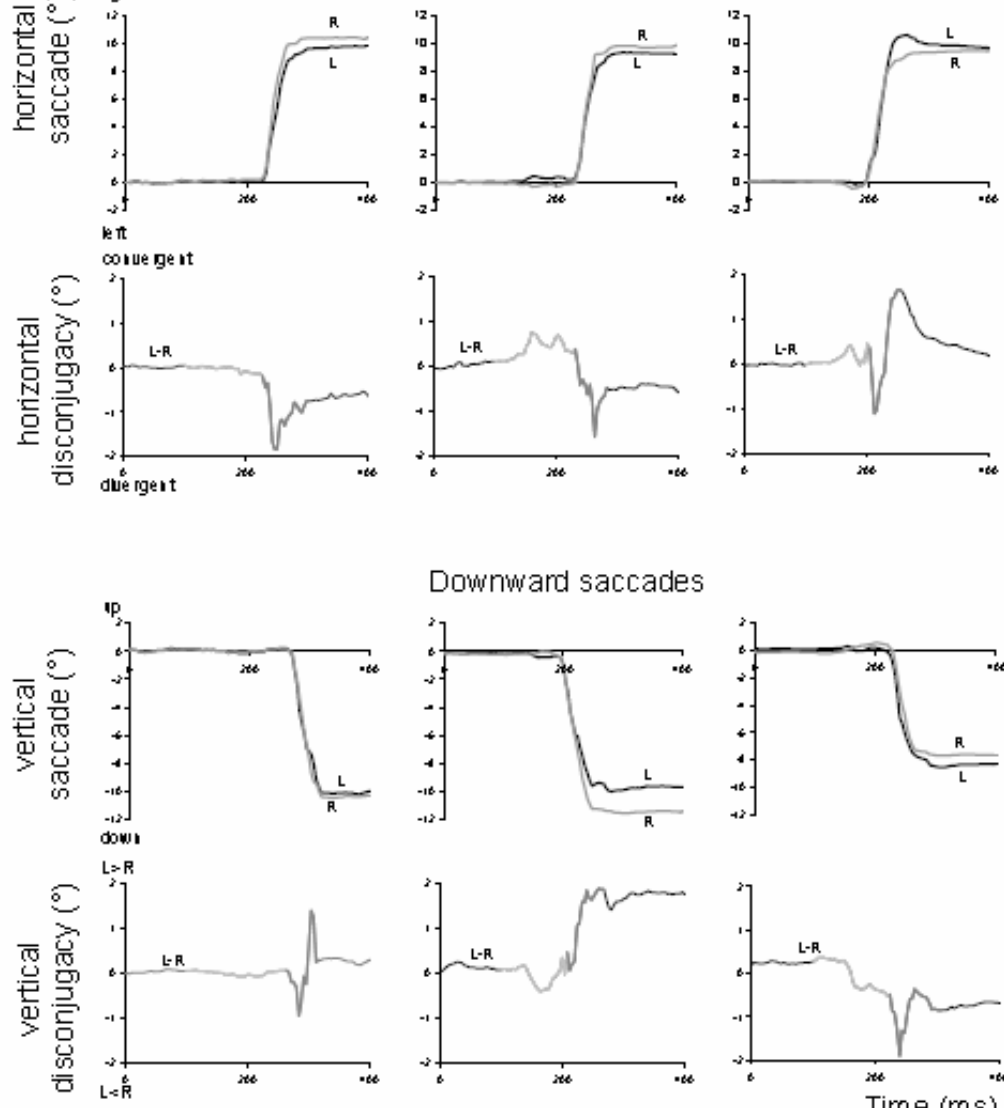

Downward saccades
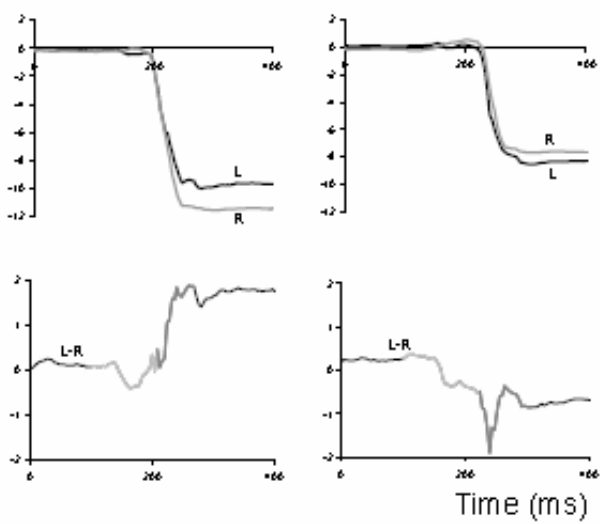

Figure 11. Sample traces during rightward (top) and downward (bottom) saccades for the no TMS condition, the left PPC stimulation and the right PPC stimulation. L: left eye; $R$ : right eye; $L-R$ : disconjugate component. The target appears at $t=0 \mathrm{~ms}$. TMS is applied at $t=100 \mathrm{~ms}$.

Vernet, Yang, Daunys, Orssaud, Eggert and Kapoula (2008) further examined this question with a paradigm of vertical and horizontal saccades of $10^{\circ}$, randomly interleaved. Single-pulse TMS was applied on the posterior parietal cortex $100 \mathrm{~ms}$ after the target onset. The TMS of the left or right PPC increased the misalignment of the eyes during the pre-saccadic fixation period and the disconjugacy of the saccades. After TMS of the right PPC, the increase of disconjugacy was significant for rightward and downward saccades, and after TMS of the left PPC, the increase was significant for downward saccades. Examination of the fixation and saccade trajectories in TMS versus no-TMS conditions (Fig. 11) did not show continuity between the perturbation of the alignment of the eyes before the saccade and the modification of the disconjugacy during the saccade.

The interpretation proposed was the existence of a vergence mechanism during the saccade, distinct from 
the mechanism used to maintain the eyes aligned during the fixation. The TMS of the PPC $100 \mathrm{~ms}$ after the target onset would disturb (i) the vergence command used to maintain the eyes aligned at the depth of fixation before the saccade and (ii) the hypothetical vergence command executed together with the saccade.

The results indicate cortical control of binocular coordination during saccades through the vergence system, in line with Hering's ideas (Hering, 1868) for the existence of two distinct systems of saccades and vergence. Existing models of saccade-vergence interaction (Busettini \& Mays, 2005; Zee et al., 1992) were originally designed to explain the interaction between saccade and vergence while shifting gaze between targets differing both in direction and in depth; the phenomenon of the acceleration of the vergence by the saccade was clearly established (Enright, 1984). Zee et al. (1992) supposed in their model a coupling between saccade and vergence subsystems that could be made through the omnipause neurons. If the omnipause neurons also partially inhibit the vergence system, the omnipause silence, allowing a saccade to occur, would also contribute to vergence acceleration. More recently, Busettini and Mays (2005) pro- posed a model in which the acceleration of the vergence by the saccade would result from a multiplicative interaction between the position signal driving the saccade system and an estimation of the vergence motor error driving the vergence system. An internal mechanism of feedback would control the movement progression, via a corticomidbrain-cortical loop. Fig. 12 shows our conceptual model aimed to explain binocular saccade coordination control. The original transient disconjugacy, caused by peripheral asymmetries at the plant level, is used as an error signal to learning and adaptation; a systematic vergence command signal is then produced together with every saccade. This command is enhanced by the interaction of saccade vergence and continuously calibrated thanks to the visual and internal feedback; the corresponding vergence is used to reduce initial disconjugacy and allows plasticity of binocular coordination. To summarize, models of eye movements in direction and in depth should be extended to consider saccade-vergence interaction as a permanent control mechanism that occurs even for saccades in a frontoparallel plane.

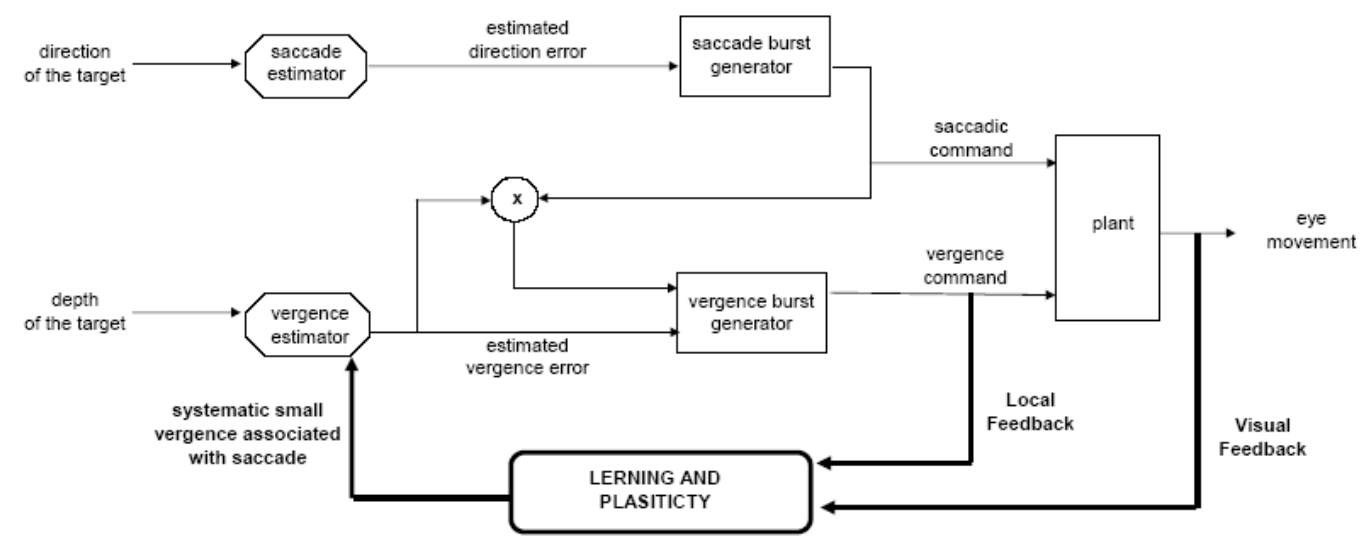

Figure 12. Conceptual model of binocular coordination of saccades using the saccade vergence interaction, inspired by models of Zee et al. (1992) and Busettini and Mays (2005). Target position is coded as direction and vergence errors (i.e. direction and vergence differences between desired position and current position). Saccade and vergence commands (pulse-slide-step, not shown) are elaborated at the brainstem (burst neurons); these commands are sent to the ocular motoneurons and orbital plant. Local feedback loops and visual feedback loops (not shown for saccade command), involving brainstem, cerebellum, and cortical areas such as PPC, allow online control of movement execution and long term calibration of the systems. Together with every saccade, a small vergence command is elaborated; the size of the vergence command is the result of prior learning and visual experience. 


\section{Concluding remarks}

The binocular coordination of saccades relies on learning and plasticity. Our hypothesis is that a vergence command is sent together with every saccade command. The CNS uses the saccade-vergence interaction substrate to prepare such intrasaccadic vergence; it aims to compensate for the peripheral asymmetries and thus to obtain a fair binocular coordination. The adaptive adjustment of the vergence command is learnt during childhood, and plasticity allows readjustments throughout life to compensate for diseases and lesions.

Learning is slower for near vision than for far vision (Yang \& Kapoula, 2003), probably because it is more difficult to adjust the size of the intrasaccadic convergence command when the eyes are already strongly converging with fixation at close distance. Learning to coordinate the saccades most likely involves a large network, including the cerebellum, the brainstem and cortical areas such as the PPC.

Fine binocular coordination underpins vision and is important in many every day tasks: reading, working on a computer screen, locating an obstacle while walking, etc. Functional problems (vertigo, dyslexia) are frequently associated with both poor vergence and poor binocular coordination of saccades (Bucci et al., 2007; Bucci et al., 2006; Bucci et al., 2004b; Kapoula et al., in press; Kapoula et al., 2007). Orthoptic vergence training in vertigo appears to improve both vergence performances and the binocular coordination of saccades (Bucci et al., 2006).

Besides their clinical implications, these studies raise important theoretical considerations. The functional problems like vertigo and dyslexia call for a less modular research approach. Study of each isolated oculomotor subsystem was a useful historical step. Such a modular approach is however not physiologically plausible and of limited clinical use, as the CNS uses large networks and many subsystems together to fulfill a function. Considering the network required for the binocular coordination of saccades, it does not appear to be an isolated problem associated with saccade command signals. Binocular coordination of saccades probably involves saccadevergence interaction, activating a large network at the cortical, brainstem and cerebellum levels. As pointed out by Ramat and Zee (2005) natural symbiosis exists between voluntary saccades, vergence, pursuit and vestibu- lar responses. The oculomotor system, being among the best understood systems in the mammalian brain, has been a model for studying theories about motor control, and for testing brain plasticity. Future oculomotor research should gain by a complex systems approach, abandoning modularity.

\section{References}

Anoh-Tanon, M.J., Bremond-Gignac, D., \& WienerVacher, S.R. (2000). Vertigo is an underestimated symptom of ocular disorders: dizzy children do not always need MRI. Pediatr Neurol, 23 (1), 49-53.

Blythe, H.I., Liversedge, S.P., Joseph, H.S., White, S.J., Findlay, J.M., \& Rayner, K. (2006). The binocular coordination of eye movements during reading in children and adults. Vision Res, 46 (22), 3898-3908.

Bucci, M.P., Bremond-Gignac, D., \& Kapoula, Z. (2007). Poor binocular coordination of saccades in dyslexic children. Graefes Arch Clin Exp Ophthalmol,

Bucci, M.P., Kapoula, Z., Bremond-Gignac, D., \& Wiener-Vacher, S. (2006). Binocular coordination of saccades in children with vertigo: dependency on the vergence state. Vision Res, 46 (21), 3594-3602.

Bucci, M.P., Kapoula, Z., Yang, Q., Bremond-Gignac, D., \& Wiener-Vacher, S. (2004a). Speed-accuracy of saccades, vergence and combined eye movements in children with vertigo. Exp Brain Res, 157 (3), 286295.

Bucci, M.P., Kapoula, Z., Yang, Q., Wiener-Vacher, S., \& Bremond-Gignac, D. (2004b). Abnormality of vergence latency in children with vertigo. J Neurol, 251 (2), 204-213.

Bucci, M.P., Paris, S., \& Kapoula, Z. (2003). Oculomotor consequences of feeble image size inequality at near reading distance. Experimental Brain Research, 149 (2), 252-259.

Busettini, C., \& Mays, L.E. (2005). Saccade-vergence interactions in macaques. II. Vergence enhancement as the product of a local feedback vergence motor error and a weighted saccadic burst. J Neurophysiol, 94 (4), 2312-2330. 
Chevrie-Muller, C., Simon, A.M., \& Fournier, S. (1997). Batterie Langage oral écrit. Mémoire. Attention (L2MA). (Paris.

Collewijn, H., Erkelens, C.J., \& Steinman, R.M. (1988a). Binocular co-ordination of human horizontal saccadic eye movements. J Physiol, 404, 157-182.

Collewijn, H., Erkelens, C.J., \& Steinman, R.M. (1988b). Binocular co-ordination of human vertical saccadic eye movements. Journal of Physiology, 404, 183-197.

Eggert, T., \& Kapoula, Z. (1995). Position dependency of rapidly induced saccade disconjugacy. Vision Research, 35 (23-24), 3493-3503.

Elkington, P.T., Kerr, G.K., \& Stein, J.S. (1992). The effect of electromagnetic stimulation of the posterior parietal cortex on eye movements. Eye, 6 ( Pt 5), 510514.

Enright, J.T. (1984). Changes in vergence mediated by saccades. Journal of Physiology, 350, 9-31.

Evans, B.J., \& Drasdo, N. (1990). Review of ophthalmic factors in dyslexia. Ophthalmic Physiol Opt, 10 (2), 123-132.

Fioravanti, F., Inchingolo, P., Pensiero, S., \& Spanio, M. (1995). Saccadic eye movement conjugation in children. Vision Res, 35 (23-24), 3217-3228.

Gamlin, P.D., \& Yoon, K. (2000). An area for vergence eye movement in primate frontal cortex. Nature, 407 (6807), 1003-1007.

Gnadt, J.W., \& Mays, L.E. (1995). Neurons in monkey parietal area LIP are tuned for eye-movement parameters in three-dimensional space. J Neurophysiol, 73 (1), 280-297.

Helmoltz, H. (1856-1866). Treatise on physiological optics. (Rochester: The Optical Society of America.

Henson, C., Staunton, H., \& Brett, F.M. (2003). Does ageing have an effect on midbrain premotor nuclei for vertical eye movements? Mov Disord, 18 (6), 688694.

Hering, E. (1868). The theory of binocular vision (translated by B. Bridgeman). (New York: Plenum Press, c1977.
Juhasz, B.J., Liversedge, S.P., White, S.J., \& Rayner, K. (2006). Binocular coordination of the eyes during reading: word frequency and case alternation affect fixation duration but not fixation disparity. Q J Exp Psychol (Colchester), 59 (9), 1614-1625.

Kapoula, Z., Bucci, M.P., Ganem, R., Poncet, S., Daunys, G., \& Bremond-Gignac, D. (in press). Free exploration of painting uncovers particular loose yoking of saccades in dyslexics. Dyslexia,

Kapoula, Z., Bucci, M.P., Jurion, F., Ayoun, J., Afkhami, F., \& Bremond-Gignac, D. (2007). Evidence for frequent divergence impairment in French dyslexic children: deficit of convergence relaxation or of divergence per se? Graefes Arch Clin Exp Ophthalmol, 245 (7), 931-936.

Kapoula, Z., Eggert, T., \& Bucci, M.P. (1995). Immediate saccade amplitude disconjugacy induced by unequal images. Vision Research, 35 (23-24), 35053518 .

Kapoula, Z., Eggert, T., \& Bucci, M.P. (1996). Disconjugate adaptation of the vertical oculomotor system. Vision Res, 36 (17), 2735-2745.

Kapoula, Z., Isotalo, E., Muri, R.M., Bucci, M.P., \& Rivaud-Pechoux, S. (2001). Effects of transcranial magnetic stimulation of the posterior parietal cortex on saccades and vergence. Neuroreport, 12 (18), 40414046.

Kapoula, Z., Yang, Q., Coubard, O., Daunys, G., \& Orssaud, C. (2004). Transcranial magnetic stimulation of the posterior parietal cortex delays the latency of both isolated and combined vergence-saccade movements in humans. Neurosci Lett, 360 (1-2), 95-99.

Kapoula, Z., Yang, Q., Coubard, O., Daunys, G., \& Orssaud, C. (2005). Role of the posterior parietal cortex in the initiation of saccades and vergence: right/left functional asymmetry. Ann N Y Acad Sci, 1039, 184197.

King, W.M., \& Zhou, W. (2002). Neural basis of disjunctive eye movements. Annals of the New York Academy of Sciences, 956, 273-283.

Kommerell, G., Olivier, D., \& Theopold, H. (1976). Adaptive programming of phasic and tonic components in saccadic eye movements. Investigations of patients with abducens palsy. Invest Ophthalmol, 15 (8), 657-660. 
Leigh, R.J., \& Zee, D.S. (2006). The neurology of eye movements. (Oxford University Press.

Lewis, R.F., Zee, D.S., Repka, M.X., Guyton, D.L., \& Miller, N.R. (1995). Regulation of static and dynamic ocular alignment in patients with trochlear nerve pareses. Vision Res, 35 (23-24), 3255-3264.

Liversedge, S.P., Rayner, K., White, S.J., Findlay, J.M., \& McSorley, E. (2006a). Binocular coordination of the eyes during reading. Curr Biol, 16 (17), 17261729.

Liversedge, S.P., White, S.J., Findlay, J.M., \& Rayner, K. (2006b). Binocular coordination of eye movements during reading. Vision Res, 46 (15), 2363-2374.

Livingstone, M.S., Rosen, G.D., Drislane, F.W., \& Galaburda, A.M. (1991). Physiological and anatomical evidence for a magnocellular defect in developmental dyslexia. Proc Natl Acad Sci U S A, 88 (18), 79437947.

Mays, L.E. (1984). Neural control of vergence eye movements: convergence and divergence neurons in midbrain. J Neurophysiol, 51 (5), 1091-1108.

Mays, L.E., \& Gamlin, P.D.R. (1995). A neural mechanism subversing saccade-vergence interactions. In: Eye Movemment Research: Mechanisms, Processes and Applications (pp. 215-223). Amsterdam: Elsevier.

Moschovakis, A.K., Scudder, C.A., \& Highstein, S.M. (1990). A structural basis for Hering's law: projections to extraocular motoneurons. Science, 248 (4959), 1118-1119.

Murshed, K.A., Ziylan, T., Seker, M., Ciekcibasi, A.E., \& Acikgozoglu, S. (2003). Morphometric assessment of brain stem and cerebellar vermis with midsagittal MRI: the gender differences and effects of age. Neuroanatomy, 2, 35-38.

Priori, A., Bertolasi, L., Rothwell, J.C., Day, B.L., \& Marsden, C.D. (1993). Some saccadic eye movements can be delayed by transcranial magnetic stimulation of the cerebral cortex in man. Brain, 116 ( Pt 2), 355367.

Ramat, S., \& Zee, D.S. (2005). Binocular coordination in fore/aft motion. Ann N Y Acad Sci, 1039, 36-53.

Robinson, F.R., Fuchs, A.F., \& Noto, C.T. (2002). Cerebellar influences on saccade plasticity. Ann N Y Acad Sci, 956, 155-163.
Stein, J., \& Walsh, V. (1997). To see but not to read; the magnocellular theory of dyslexia. Trends Neurosci, 20 (4), 147-152.

Stein, J.F., Riddell, P.M., \& Fowler, M.S. (1987). Fine binocular control in dyslexic children. Eye, 1 ( Pt 3), 433-438.

Stein, J.F., Riddell, P.M., \& Fowler, S. (1988). Disordered vergence control in dyslexic children. Br J Ophthalmol, 72 (3), 162-166.

Straube, A., Deubel, H., Ditterich, J., \& Eggert, T. (2001). Cerebellar lesions impair rapid saccade amplitude adaptation. Neurology, 57 (11), 2105-2108.

Takagi, M., Tamargo, R., \& Zee, D.S. (2003). Effects of lesions of the cerebellar oculomotor vermis on eye movements in primate: binocular control. Prog Brain Res, 142, 19-33.

Tian, S., Nishida, Y., Isberg, B., \& Lennerstrand, G. (2000). MRI measurements of normal extraocular muscles and other orbital structures. Graefes Arch Clin Exp Ophthalmol, 238 (5), 393-404.

Tzelepi, A., Lutz, A., \& Kapoula, Z. (2004). EEG activity related to preparation and suppression of eye movements in three-dimensional space. Exp Brain Res, 155 (4), 439-449.

Tzelepi, A., Yang, Q., \& Kapoula, Z. (2005). The effect of transcranial magnetic stimulation on the latencies of vertical saccades. Exp Brain Res, 164 (1), 67-77.

van der Steen, J., \& Bruno, P. (1995). Unequal amplitude saccades produced by aniseikonic patterns: effects of viewing distance. Vision Research, 35 (23-24), 34593471.

Vernet, M., Yang, Q., Daunys, G., Orssaud, C., Eggert, T., \& Kapoula, Z. (2008). How the Brain Obeys Hering's Law: A TMS Study of the Posterior Parietal Cortex. Invest Ophthalmol Vis Sci, 49 (1), 230-237.

Versino, M., Hurko, O., \& Zee, D.S. (1996). Disorders of binocular control of eye movements in patients with cerebellar dysfunction. Brain, 119, 1933-1950.

Yang, Q., \& Kapoula, Z. (2003). Binocular coordination of saccades at far and at near in children and in adults. J Vis, 3 (8), 554-561.

Yang, Q., \& Kapoula, Z. (2004). TMS over the left posterior parietal cortex prolongs latency of contralateral saccades and convergence. Invest Ophthalmol Vis Sci, 45 (7), 2231-2239. 
Yang, Q., \& Kapoula, Z. (2006). Aging does not affect the accuracy of vertical saccades nor the quality of their binocular coordination: A study of special elderly group. Neurobiol Aging,

Ygge, J., Lennerstrand, G., Axelsson, I., \& Rydberg, A. (1993). Visual functions in a Swedish population of dyslexic and normally reading children. Acta Ophthalmol (Copenh), 71 (1), 1-9.

Ygge, J., Lennerstrand, G., Rydberg, A., Wijecoon, S., \& Pettersson, B.M. (1993). Oculomotor functions in a Swedish population of dyslexic and normally reading children. Acta Ophthalmol (Copenh), 71 (1), 10-21.

Ygge, J., \& Zee, D.S. (1995). Control of vertical eye alignment in three-dimensional space. Vision Res, 35 (22), 3169-3181.

Zee, D.S., Fitzgibbon, E.J., \& Optican, L.M. (1992). Saccade-vergence interactions in humans. J Neurophysiol, 68 (5), 1624-1641.

Zhou, W., \& King, W.M. (1998). Premotor commands encode monocular eye movements. Nature, 393 (6686), 692-695.

American Psychological Association. (2001). Publication Manual of the American Psychological Association (5th ed.). Washington, DC: Author.

American Psychological Association. (2005). Concise Rules of APA Style. Washington, DC: Author.

Houghton, P. M., Houghton, T. J., \& Peters, M. F. (2005). APA: The Easy Way!. Port Huron, MI: Baker College. 